\title{
Design and Implementation of Low Density Parity Check Codes
}

\author{
S.A.Sivasankari \\ Assistant professor, Saveethaschool of engineering, Saveethauniversity, Thandalam, Chennai-602105.
}

\begin{abstract}
LDPC is one of the best in coding theory nowadays. In this paper I Design and Implement this LDPC codes in Xilinx VHDL platform. I achieved two results one for data sending with error and make correction and send without error. This code which helps in both communication based transmission as well as data based binary transmission. Here all the concepts are known by the information theory and coding techniques. This paper which shows how the data's are sending from transmitter to receiver and resend again by correcting the same.
\end{abstract}

Keywords: - Tanner graphs, Bipartite graphs, $V_{-}$nodes, $C_{-}$nodes, LDPC decoder, Hard decision decoding, Soft decision decoding.

\section{INTRODUCTION}

Low density parity check codes are popularly known as LDPC codes. These codes are proposed by Galleger at MIT in the year of 1962. These codes are known as Galleger codes. But these codes are neglected very much for this past 35 years. These codes are the linear error correcting codes which are used in information theory. These are the class of linear block codes. The name of this code arrives from parity- check matrix concept which has only few one's when compared with zeros. Nowadays parallel architecture is in use. Thus these codes are suited for implementation of current scenario. The forward error correction codes are dominated well on those days due to highly structured algebraic block and convolutional codes. Here, the use of sparse matrix, turbo codes, Tanner graphs are enormously comes in to picture. Nowadays LDPC codes are used in Wi$\max$ for microwave communications, $\mathrm{CMMB}$ i.e. china multimedia mobilebroadcasting, Digital video broadcasting and for Wi-Fi standard.

\subsection{Representation of LDPC codes}

LDPC codes are represented as matrix based or graph based. First Section which is based on matrices and second section based on graphs.

\subsection{Matrix representation}

Matrix defined is the parity check matrix with the dimension of $(8,4)$ code i.e. $\left(n^{*} \mathrm{~m}\right)$.Wr which is number of one's in row and Wc is the number of ones in columns. The low density matrix to be satisfied the conditions as $\mathrm{W}_{\mathrm{c}}<<\mathrm{n}$ and $\mathrm{Wr}<<\mathrm{m}$. [2]

Fig: 1 Parity check matrix H matrix[2]

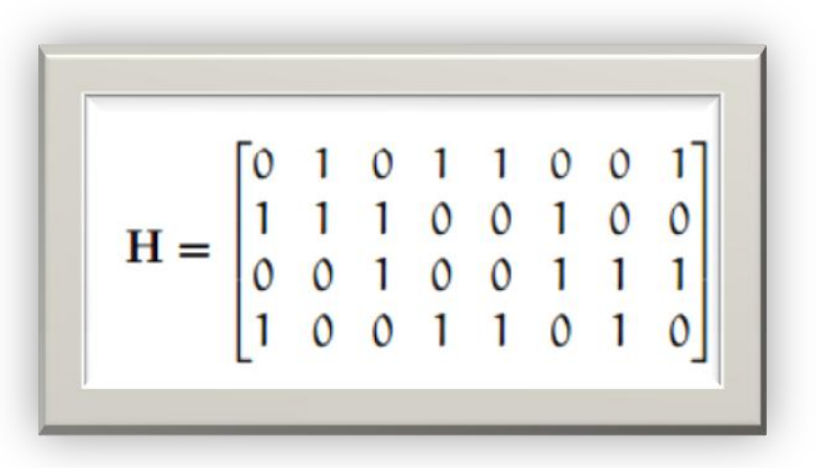

1.3 Graphical representation

Fig : 2 Tanner graphs[2] 


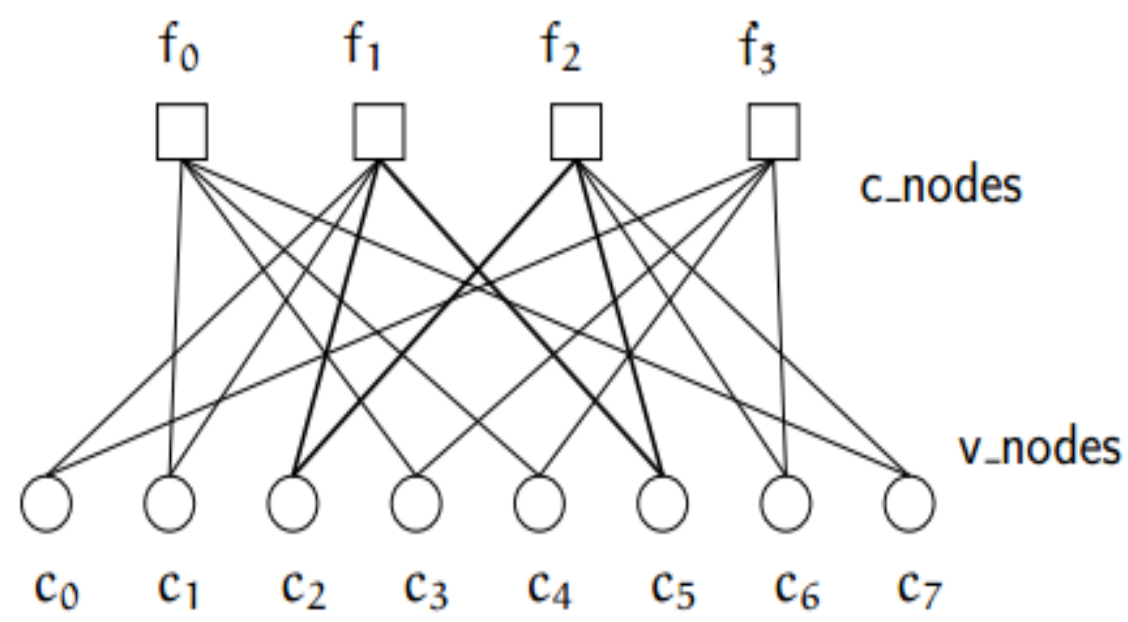

Consider above parity matrix as representation for this graph shown above. Tanner who introduced this matrix for LDPC codes. The main advantage of this graph is that it shows the full and complete representation of the code and also describes the decoding algorithm going to be used on rest of the paper.

Tanner graphs are bipartite graphs. Bipartite graph means that whose vertices are divided in to two disjoint sets .These are independent sets. Thus there will be connectivity between two disjoint sets. Thus the two types of nodes in Tanner graph are named as $\mathrm{C}_{-}$nodes and $\mathrm{V}_{-}$nodes. There is no connectivity between $\mathrm{C}_{0}$ and $\mathrm{F}_{0}$ thence the first place is having 0.1 in the second place means that connectivity lies between $\mathrm{F}_{0}$ and $\mathrm{C}_{1}$.

Check nodes are specified as m nodes that are number of parity bits and variable nodes are $\mathrm{n}$ which are known as number of bits in code word. Thus the element of $\mathrm{H}$ matrix that is parity matrix is 1 there is connectivity between $\mathrm{F}_{\mathrm{i}}$ and $\mathrm{C}_{\mathrm{j}}$.

\subsection{Error correction codes using parity -checks}

Here all the communications are based on bits 0's and 1's.Hence transmitted messages and received messages are 0 's and 1's. For the forward error control coding techniques to the augmented message bits have to introduce the redundancy bits that are check bits to produce a code word for the particular message. Hence for every message there is a different code word in such a way a message sent from transmitter and the message received by receiver can be ensured.

The coding scheme discussed here is SPC. Single parity check code which involves the addition of a single extra bit to the binary message and that value which depends upon the message bits.

\subsection{Regular and irregular LDPC codes}

There are two types of LDPC codes as regular and irregular. If $\mathrm{W}_{\mathrm{c}}$ is constant for every column and $\mathrm{W}_{\mathrm{r}}$ and $\mathrm{W}_{\mathrm{C}}$ are equal as well as $\mathrm{n} / \mathrm{m}$ is constant for every row. There is regularity at the graphical representation. There are same numbers of edges lies between $\mathrm{C}_{-}$nodes and $\mathrm{V}_{-}$nodes. This type is a regular LDPC codes.

If the numbers of 1's are not constant in each row and column then that code is termed as Irregular LDPC codes.

\section{LDPC ALGORITHMS}

There are different algorithms for both encoding and decoding. The most common algorithms for decoding are Belief propagation algorithms, Message passing algorithms as sum-product algorithms. There is one more decoding algorithm which is hard- decision decoding algorithm.

\subsection{Encoding}

Decoding algorithm is applicable only for error correction. This is opt for traditional systematic block code. Thus code word consists of the message and some parity check bits. One possible and advantageous in the LDPC codes is that we can directly see the original message bits in a encoded LDPC message.

\subsection{Performance and complexity}

The feature of the LDPC codes which are based on Shannon limit. The year 1948 is information theory's birth. That time ClaudeE.Shannonpublished his paper on the limits of reliable transmission of data over unreliable channels. As well as he showed the methods how to achieve these limits. The interesting fact is that the high performance codes are irregular. Thelarge block length which results in large parity check and generator 
matrices. The complexity of multiplying a codeword with matrix which depends on the amount of 1's in the matrix. The sparse matrix $\mathrm{H}$ which is denoted in the form $\left[\mathrm{P}^{\mathrm{T}} \mathrm{I}\right]$. The Generator matrix $\mathrm{G}$ can be calculated as $\mathrm{G}=[\mathrm{I}] \mathrm{P}]$ where $\mathrm{P}$ is the sub- matrix which is not a sparse matrix and hence the encoding complexity is quite high. The complexity which affects the sparse matrix. The best performance will never achieve if the block length gets very high. Hence there's a need of iterative decoding algorithms. Those type of algorithms which perform local calculations and pass those results via messages.

\subsection{Decoding}

The algorithm used to decode LDPC codes which was discovered independently several times [2].The most common used algorithms are the belief propagation algorithm, the message passing algorithm and the sumproduct algorithm [2].There are two types of decision decoding as hard decision decoding and soft decision decoding which are described below.

\subsection{Hard- decision decoding}

Let's consider that we have a BHC channel and the received code word with one error bit.

In the first step all V-nodes send a message to $\mathrm{C}$ - nodes believe that there is no error. At this stage the bits present at $\mathrm{V}$ - nodes will received by $\mathrm{C}$ - nodes.

In the second step every check nodes calculate a response to every connected variable node.

In the third step the $\mathrm{V}$-nodes receive the messages from check nodes and use the additional information to decide if the original receive bit is not an error.

For the verification we can consider that $\mathrm{V}$ - node has three sources of information i.e. the original bit and two suggestions from check nodes [2].

The main advantage of this hard-decision decoding is that it corrects the transmission error rate.

\subsection{Soft-decision decoding}

Soft-decision decoding of LDPC codes which is based on belief propagation algorithm. This yields better decoding performance. This is actually preferred method. The idea is exactly same as in hard-decision decoding. In this method the message sent by the variable node to the check node. Every message contains in pairs always which stands for amount of belief. This soft- decision decoding algorithm is very simple variant suited for BSC channels. This is best one for performance improvements. The results will come close to zero for large block lengths.

\subsection{Error detection and correction}

Suppose a code word sent from transmitting section to receiving section which gets flipped. In LDPC codes it is easily possible to detect and correct the flipped bit. Every code word should satisfy the equation given below. If this equation is not satisfied then we should know that the received bits are error.

The vector $\mathrm{s}=\mathrm{Hy}^{\mathrm{T}}$ which specifies the syndrome of $\mathrm{y}$.

$$
\mathrm{Hy}^{\mathrm{T}}=0 \quad \text { [3] where } \mathrm{H} \text { represents parity check matrix. }
$$

The numbers of bit positions differ between two code words which are termed as HAMMING DISTANCE.Minimum hamming distance is the measure of ability of the code to detect error.

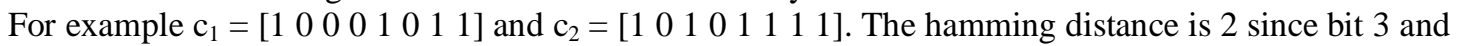
bit 6 gets flipped.

\section{LDPC CONSTRUCTION}

Binary LDPC codes construction involves which assigns small number of 1's in all zero matrixes. It needs all rows and columns to be required degree of distribution. There are three different types of LDPC construction designed by Gallager, proposed by McKay and Neal and repeat accumulation code methods.

Actually the original LDPC codes which are presented by Gallager which are regular and defined by H.Here each row and column is divided in such a way that there are equal number of 1 's in consecutive rows and columns. [3].

$$
H=\left[\begin{array}{llllllllllll}
1 & 1 & 1 & 1 & 0 & 0 & 0 & 0 & 0 & 0 & 0 & 0 \\
0 & 0 & 0 & 0 & 1 & 1 & 1 & 1 & 0 & 0 & 0 & 0 \\
0 & 0 & 0 & 0 & 0 & 0 & 0 & 0 & 1 & 1 & 1 & 1 \\
\hline 1 & 0 & 1 & 0 & 0 & 1 & 0 & 0 & 0 & 1 & 0 & 0 \\
0 & 1 & 0 & 0 & 0 & 0 & 1 & 1 & 0 & 0 & 0 & 1 \\
0 & 0 & 0 & 1 & 1 & 0 & 0 & 0 & 1 & 0 & 1 & 0 \\
\hline 1 & 0 & 0 & 1 & 0 & 0 & 1 & 0 & 0 & 1 & 0 & 0 \\
0 & 1 & 0 & 0 & 0 & 1 & 0 & 1 & 0 & 0 & 1 & 0 \\
0 & 0 & 1 & 0 & 1 & 0 & 0 & 0 & 1 & 0 & 0 & 1
\end{array}\right]
$$

Fig 3: REGULAR GALLAGER PARITY CHECK MATRIX[3] 
Another popular method of LDPC code constructions are proposed by McKay and Neal.Here the columns of one gets added with another column in such a way that the number of 1 'sin row and column which are correct bit distribution.

Fig 4: Mckay and Neal parity check matrix Regular[3]

$$
H=\left[\begin{array}{llllllllllll}
1 & 0 & 0 & 0 & 0 & 1 & 0 & 1 & 0 & 1 & 0 & 0 \\
1 & 0 & 0 & 1 & 1 & 0 & 0 & 0 & 0 & 0 & \mathbf{1} & 0 \\
0 & 1 & 0 & 0 & 1 & 0 & 1 & 0 & 1 & 0 & 0 & 0 \\
0 & 0 & 1 & 0 & 0 & 1 & 0 & 0 & 0 & 0 & \mathbf{1} & 1 \\
0 & 0 & 1 & 0 & 0 & 0 & 1 & 1 & 0 & 0 & 0 & 1 \\
0 & 1 & 0 & 0 & 1 & 0 & 0 & 0 & 1 & 0 & \mathbf{1} & 0 \\
1 & 0 & 0 & 1 & 0 & 0 & 1 & 0 & 0 & 1 & 0 & 0 \\
0 & 1 & 0 & 0 & 0 & 1 & 0 & 1 & 0 & 1 & 0 & 0 \\
0 & 0 & 1 & 1 & 0 & 0 & 0 & 0 & 1 & 0 & 0 & 1
\end{array}\right]
$$

Another type or method of LDPC codes which are termed as repeat- accumulate codes which the structure makes the repeat accumulate codes systematic and allows them to be easily encoded.

$$
H=\left[\begin{array}{llllllllllll}
1 & 0 & 0 & 1 & 0 & 0 & 0 & 0 & 0 & 0 & 0 & 0 \\
1 & 0 & 0 & 1 & 1 & 0 & 0 & 0 & 0 & 0 & 0 & 0 \\
0 & 1 & 0 & 0 & 1 & 1 & 0 & 0 & 0 & 0 & 0 & 0 \\
0 & 0 & 1 & 0 & 0 & 1 & 1 & 0 & 0 & 0 & 0 & 0 \\
0 & 0 & 1 & 0 & 0 & 0 & 1 & 1 & 0 & 0 & 0 & 0 \\
0 & 1 & 0 & 0 & 0 & 0 & 0 & 1 & 1 & 0 & 0 & 0 \\
1 & 0 & 0 & 0 & 0 & 0 & 0 & 0 & 1 & 1 & 0 & 0 \\
0 & 1 & 0 & 0 & 0 & 0 & 0 & 0 & 0 & 1 & 1 & 0 \\
0 & 0 & 1 & 0 & 0 & 0 & 0 & 0 & 0 & 0 & 1 & 1
\end{array}\right]
$$

Fig : 4. REPEAT-ACCUMULATE CODES.

\section{IV.}

APPLICATIONS

It is error correcting codes in DVB-s2 standard for satellite communication for digital television. It is also used in Ethernet 10 base T. It is also a part of Wi-Fi 802.11 standard. The optional parts of it are 802.11 ac and $802.11 \mathrm{n}$. It is also used in OFDM networks where data transmission to be without error. It is even with low bit rate also.

\section{RESULTS}

Here it is implemented and simulated via VHDL.Based on that simulation result which achieved as the graphical form. The tools used are Xilinx software as well as modelsim software.

\section{SIMULATION}

\section{WITH OUT CORRECTION}

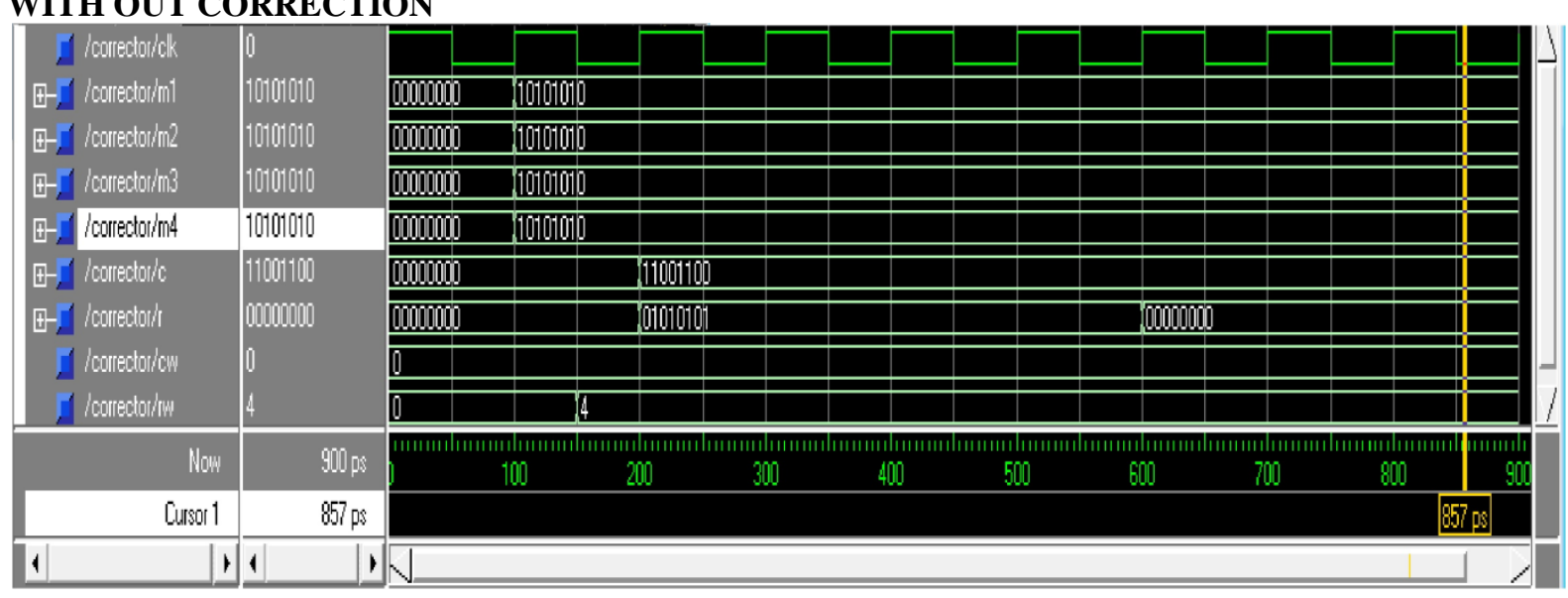




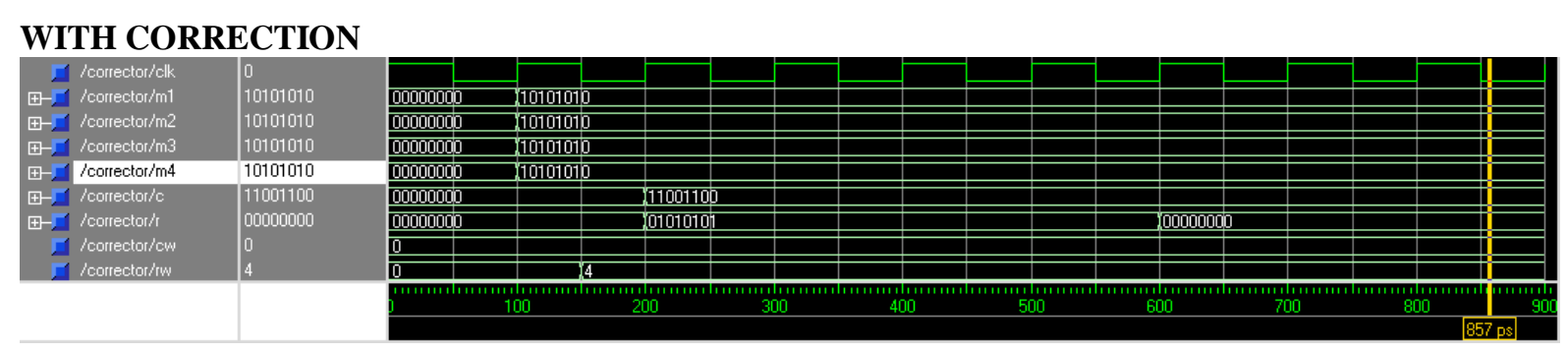

\section{SUMMARY}

Iterative decoding approach is already used in turbo codes .But the LDPC codes which gives better results. Here code rate may be higher. But error rate is low. This may be possible to implement parallelizable decoders. The main disadvantages of this LDPC codes are more complex and long code length only yields better results. There are many different classes of codes LDPC codes already equipped with very fast encoding and decoding algorithms. By this algorithm it is possible to recover the original code word

\section{ACKNOWLEDGEMENT}

I am sincerely giving my thanks to my Guide Dr.D.Dhanasekaran, Head of the department Dr. P. C. Kishore raja and many thanks to My son Appu and who helped to complete this journal.

\section{REFERENCES}

[1] Amin shokrollahi, "LDPC codes", Digital Fountain, Inc.

[2] Bernhard M.J. Leiner, "LDPC codes“, April 8, 2005

[3] Sarah Johnson, "Introducing low density parity check codes ", ACoRN Spring School version 1.1

[4] C.E.Shannon,"A mathematical theory of communications" Bell system Technical journal,

[5] vol 27,pp 379-423,1948.

[6] B.Bollobas, External graph theory. Academic press, 1978. 